\title{
The second dose of the BNT162b2 mRNA vaccine does not boost SARS-CoV-2 neutralizing antibody response in previously infected subjects
}

\author{
Ilaria Vicenti ${ }^{1} \cdot$ Francesca Gatti $^{2} \cdot$ Renzo Scaggiante $^{3} \cdot$ Adele Boccuto $^{1} \cdot$ Daniela Zago ${ }^{2} \cdot$ Monica Basso $^{2}$. \\ Filippo Dragoni ${ }^{1}$ - Saverio Giuseppe Parisi ${ }^{2}$ Maurizio Zazzi ${ }^{1}$
}

Received: 30 May 2021 / Accepted: 30 July 2021 / Published online: 3 August 2021

(c) Springer-Verlag GmbH Germany, part of Springer Nature 2021

While SARS-CoV-2 vaccination of uninfected hosts is proceeding worldwide, whether and how to vaccinate those recovered from natural infection remains debatable. A single-dose mRNA vaccine administration has been reported to boost SARS-CoV-2 neutralizing antibodies (NtAb) in subjects with past infection $[1,2]$ while the effect of a second dose in such subjects has not been documented. Within a health care worker (HCW) surveillance and vaccination program running at a SARS-CoV-2 referral hospital, we assessed the dynamics of SARS-CoV-2 NtAb in 22 uninfected and 34 previously infected individuals who completed the 2-dose schedule of the BNT162b2 mRNA vaccine. All the subjects received the second dose vaccine 3 weeks after the first dose. The subjects included in the uninfected group had no SARS-COV-2 RNA detection in the tests performed according to the hospital surveillance program and at the time of NtAb testing. In addition, all the subjects had been tested for the presence of a neutralizing antibody titer at baseline, i.e., at the time of the first vaccine administration, and confirmed as negative.

$\mathrm{NtAb}$ titers were determined in a live virus assay in VERO E6 cells infected by a lineage B.1 isolate and defined as the reciprocal of the serum dilution decreasing virus replication by $50 \%\left(\mathrm{EC}_{50}\right)$, as measured by the CellTiter-Glo ${ }^{\circledR}$ Luminescent Cell Viability Assay (Promega) [3].

Saverio Giuseppe Parisi and Maurizio Zazzi equally contributed to the manuscript and should be considered as shared last authors.

Saverio Giuseppe Parisi

saverio.parisi@unipd.it

1 Department of Medical Biotechnologies, University of Siena, Siena, Italy

2 Department of Molecular Medicine, University of Padova, Via Gabelli 63, 35121 Padova, Italy

3 Belluno Hospital, Belluno, Italy
A written informed consent was obtained from all the $\mathrm{HCW}$ to be enrolled in a NtAb follow-up study, as approved by the comitato per la sperimentazione clinica di Treviso e Belluno and in accordance with the ethical standards as laid down in the 1964 Declaration of Helsinki and its later amendments.

$\mathrm{NtAb}$ titers were expressed as median (IQR) and the non-parametric Wilcoxon Signed Rank Sum test and Mann-Whitney test were used to analyze changes in paired and unpaired data, respectively. Analyses were run by IBM SPSS Statistics, version 20, and all p values were two-sided.

The previously infected subjects were $32.4 \%$ males, had median [IQR] age 44 [33-52] years and recovered from asymptomatic or mild infection without hospitalization during the first wave of the epidemic in March and April 2020. Sera for SARS-CoV-2 NtAb titers measurement before first-dose vaccination (T0, January or February 2021) were obtained at median 293.0 (265.7-301.0) days after SARS$\mathrm{CoV}-2$ diagnosis. In this group, median NtAb titers significantly increased 21 days post first-dose vaccination (T1) (1421.5 [700.0-2317.0]) and 21 days post second-dose vaccination (T2) (1695.5 [878.4-2768.7]) with respect to T0 (28.0 [5.0-120.5]; $p<0.001$ for both comparisons). The increase at $\mathrm{T} 2$ vs. T1 was still significant $(p=0.018)$, however the T1/T0 fold-change was much higher than the T2/T1 fold change (33.0 [8.8-80.5] vs. $1.5[1.1-2.0] ; p<0.001)$. In the uninfected group (27.3\% males, median age 49 [38-59] years), $\mathrm{NtAb}$ titers at $\mathrm{T} 2$ also increased significantly with respect to $\mathrm{T} 1(171.0[100.7-230.1]$ vs. 6.5 [5.0-15.2]; $p=0.007)$. Thus, the T2/T1 fold-change was higher in the uninfected group compared with the previously infected group $(p<0.001)$ while the T1/T0 fold-change in the previously infected group was similar to the T2/T1 fold-change in the uninfected group $(p=0.084)$. NtAb titers obtained from each data set are indicated in Fig. 1. 
Fig. 1 SARS-CoV-2 neutralizing antibody $(\mathrm{NtAb})$ titers in subjects with past SARS-CoV-2 infection measured at vaccination baseline (T0), 21 days after first-dose vaccination (T1) and 21 days after seconddose vaccination and $\mathrm{NtAb}$ titers measured at vaccination baseline (T0), 21 days after first-dose vaccination (T1) and 21 days after second-dose vaccination in subjects without past SARS-CoV-2 infection. The same symbols indicate the same subjects at different time points. Paired data were analyzed by Wilcoxon Signed Ranks Test. Asterisks indicate significance levels: *** $p<0.001 ; * * p<0.01$; $* p<0.05$

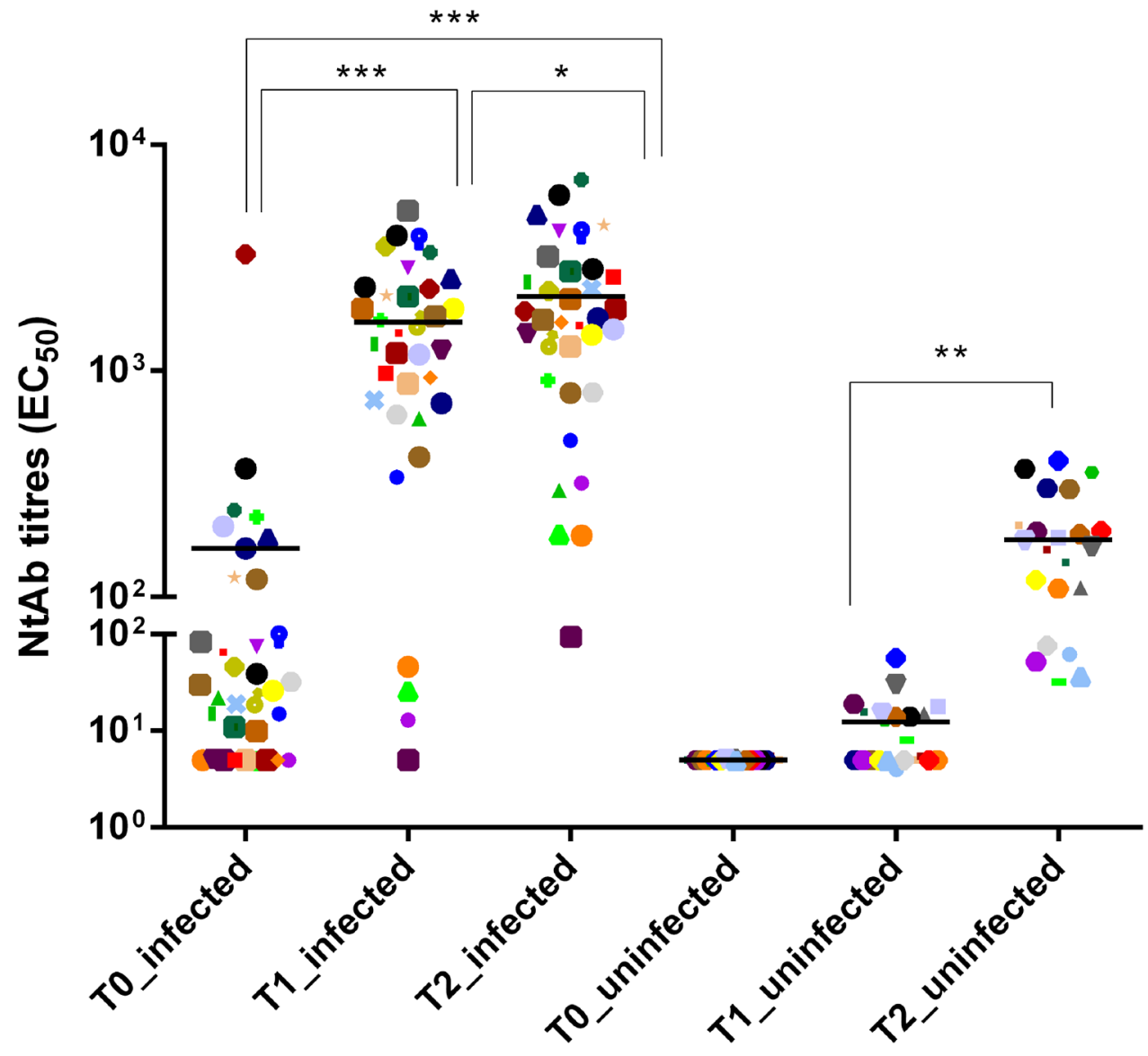

This data do not support the need for a second dose of BNT162b2 vaccine in subjects previously infected with SARS-CoV-2, given the minimal further increase of NtAb achieved. A significant boost of naturally acquired immunity occurs with the first dose, although it remains to be established whether this increase translates into improved protection from reinfection or simply reflects the secondary immune response to recall antigen which would be protective per se. While the previously infected group in this study had asymptomatic or mild SARS-CoV-2 infection, subjects experiencing more symptoms are expected to develop higher NtAb titers on their own [4], thus a benefit from a second dose of vaccine is even more unlikely for this patient population. Strengths of the study are the test used (an authentic virus neutralization with a SARSCoV-2 isolate circulating in Italy at $\mathrm{HCW}$ enrollment), the homogeneity of the study population and the very long interval, a median of 10 month, from recovery to $\mathrm{NtAb}$ titers measurement before first-dose vaccination. However, vaccine recall could be an option when a long time has passed since natural infection because NtAb titers consistently decline over time [5] and their duration at protective levels remains to be determined.
Acknowledgements We would like to thank Alessia Lai (University of Milan) for making the SARS-CoV-2 lineage B strain available for this study.

Author contributions Concept and design: SGP. Acquisition, analysis, or interpretation of data: all authors. Drafting of the manuscript: SGP, MZ. Critical revision of the manuscript for important intellectual content: SGP, MZ, IV. Statistical analysis: MZ, IV. Administrative, technical, or material support: DZ, MB. Supervision: MZ, SGP.

Funding This work was supported by University of Padua, grant number DOR-2019 and DOR-2020 to SGP.

Availability of data and material Raw data can be made available by the corresponding author upon reasonable request.

Code availability Not applicable.

\section{Declarations}

Conflict of interest None to declare.

Ethical approval The study was approved by the comitato per la sperimentazione clinica di Treviso e Belluno and it was performed in accordance with the ethical standards as laid down in the 1964 Declaration of Helsinki and its later amendments. 
Consent to participate A written informed consent was obtained from all the participants.

Consent for publication Not applicable.

\section{References}

1. Krammer F, Srivastava K, Alshammary H, Amoako AA, Awawda $\mathrm{MH}$, Beach KF, et al. Antibody responses in seropositive persons after a single dose of SARS-CoV-2 mRNA vaccine. N Engl J Med. 2021;384:1372-4. https://doi.org/10.1056/NEJMc2101667.

2. Saadat S, Rikhtegaran Tehrani Z, Logue J, Newman M, Frieman MB, Harris AD, et al. Binding and neutralization antibody titers after a single vaccine dose in health care workers previously infected with SARS-CoV-2. JAMA. 2021;325:1467-9. https://doi. org/10.1001/jama.2021.3341.

3. Vicenti I, Gatti F, Scaggiante R, Boccuto A, Zago D, Basso M, et al. Single-dose BNT162b2 mRNA COVID-19 vaccine significantly boosts neutralizing antibody response in health care workers recovering from asymptomatic or mild natural SARS-CoV-2 infection. Int J Infect Dis. 2021;108:176.

4. Dufloo J, Grzelak L, Staropoli I, Madec Y, Tondeur L, Anna F, et al. Asymptomatic and symptomatic SARS-CoV-2 infections elicit polyfunctional antibodies. Cell Rep Med. 2021;2: 100275. https://doi.org/10.1016/j.xcrm.2021.100275.

5. Muecksch F, Wise H, Batchelor B, Squires M, Semple E, Richardson $\mathrm{C}$, et al. Longitudinal serological analysis and neutralizing antibody levels in coronavirus disease 2019 convalescent patients. J Infect Dis. 2021;223:389-98. https://doi.org/10.1093/infdis/jiaa6 59. 